\title{
Investigating and improving introductory physics students' understanding of electric field and the superposition principle: The case of a continuous charge distribution
}

\author{
Jing Li and Chandralekha Singh \\ Department of Physics and Astronomy, University of Pittsburgh, Pittsburgh, Pennsylvania 15260, USA
}

(Received 13 May 2018; published 27 February 2019)

\begin{abstract}
We discuss an investigation of the difficulties that students in a calculus-based university introductory physics course have with electric field and the superposition principle for the case of a continuous charge distribution and how that research was used as a guide in the development, validation, and evaluation of a tutorial on these topics to help students learn these concepts. The tutorial uses a guided inquiry-based approach to learning and involved an iterative process of development and validation. During the validation process, we obtained feedback both from physics instructors who regularly teach introductory courses in which these concepts are taught and from students for whom the tutorial is intended. Then the final version of the tutorial was administered in several sections of a calculus-based introductory physics course after traditional lecture-based instruction in relevant concepts. We discuss the performance of students in individual interviews and on the pretest administered before the tutorial (but after traditional lecture-based instruction) and on the post-test administered after the tutorial in three sections of the introductory physics course. We also compare student performance in several sections of the course in which students worked on the tutorial with another section in which students only learned the material via traditional lecture-based instruction. We find that students who used the tutorial performed significantly better compared to those who learned the material only via traditional lecture-based instruction.
\end{abstract}

DOI: 10.1103/PhysRevPhysEducRes.15.010116

\section{INTRODUCTION}

Electrostatics is an important topic in most calculusbased second-semester introductory physics courses. Although Coulomb's law and the superposition principle are taught extensively in a majority of these courses for science and engineering majors, these concepts are challenging for many students after traditional lecture-based instruction. Despite the fact that students may have learned the superposition principle in the context of forces in introductory mechanics, this learning does not automatically transfer from mechanics to the more abstract context of electrostatics and students often struggle in applying the superposition principle due to the different "surface" features of the electrostatics problems compared to mechanics problems [1-2]. Finding the net electric field due to a charge distribution, discrete or continuous, requires understanding the principle of superposition for the electric fields and students must learn to add the field vectors at a point due to various charges in the region. Moreover, the difficulties with the conceptual and procedural knowledge

Published by the American Physical Society under the terms of the Creative Commons Attribution 4.0 International license. Further distribution of this work must maintain attribution to the author(s) and the published article's title, journal citation, and DOI. become compounded in the context of a continuous charge distribution. Since these concepts are challenging, developing research-validated learning tools to help students learn these concepts can help them develop a more coherent knowledge structure and also improve students' problem solving and reasoning skills [1-15].

Investigation of student difficulties related to electricity and magnetism concepts is important for designing instructional strategies to reduce the difficulties and help students develop a good grasp of relevant concepts. Here we discuss an investigation of student difficulties with the electric field and the superposition principle for the case of a continuous charge distribution and how that research on student difficulties was used as a guide in the development and evaluation of a tutorial to help introductory physics students develop a functional understanding of these concepts. We find that students in the sections of the course in which the tutorial was used performed significantly better on the post-test than those in the section of the course in which students did not engage with the tutorial.

\section{PRIOR INVESTIGATIONS IN INTRODUCTORY ELECTRICITY AND MAGNETISM}

Several prior studies by several groups have focused on the difficulties of introductory physics students with electricity and magnetism and strategies that may help students learn these concepts better [16-35]. For example, 
McDermott, Shaffer, and the Physics Education Group at University of Washington have performed investigations of the difficulties students have with learning introductory physics concepts and developed an inquiry-based curriculum on various topics, e.g., electrical circuits, that focuses on significantly reducing the difficulties [16-18]. The investigation by Eylon and Ganiel suggests that macro-micro relationships may be the missing link between electrostatics and electrodynamics in students' reasoning [19]. Gladding et al. have developed a smartPhysics suite focused on helping students learn electricity and magnetism [20]. Zuza et al. have investigated student difficulties with Faraday's law and strategies to help students learn related concepts better [21-22]. Guruswamy et al. [23] studied student understanding of transfer of charge between conductors, and Guisasola et al. [24-26] have carried out investigations related to student understanding of capacitors. Dębowska et al. reported on robust multimedia resources to help students learn electricity and magnetism [27]. Savelsbergh et al. have investigated the role of situational knowledge in learning electricity and magnetism [28]. Sujarittham et al. [29] have developed guided active learning worksheets while Efthimiou et al. [30] and Lenaerts et al. [31] have used pedagogical approaches to help students learn, e.g., by using peer instruction. Itza-Ortiz et al. studied students' models of Newton's second law in mechanics and electromagnetism and found that three different models were prevalent in student reasoning [32]. Alejandro and Zavala have contrasted students' understanding of electric field and electric force [33]. Sokoloff and Thornton have developed interactive lecture demonstrations, many of which focus on electricity and magnetism concepts [34]. Thacker et al. investigated student understanding of transients in direct current electric circuits [35]. Singh and collaborators have also investigated student reasoning difficulties with electrical circuit elements, nonidentical light bulbs in series and parallel, electric field and superposition principle, electric flux, Gauss's law, conductors and insulators, as well as the role of peer instruction and role of representation in learning electrostatics [36-50]. Several research-based assessment tools that focus on introductory electricity and magnetism have been developed [51-59]. For example, Maloney et al. [52] and Ding et al. [53] developed tests that broadly survey many important concepts covered in the introductory physics courses. Engelhard et al. [54] have developed a conceptual assessment related to circuits, Singh and Li [55-57] a conceptual assessment on magnetism, and Singh a conceptual assessment on symmetry and Gauss's law [58-59]. These surveys show that introductory students have conceptual difficulties with fundamental concepts related to electricity and magnetism.

\section{METHODOLOGY}

The students who participated in this investigation were enrolled in different "equivalent" sections of a second semester college introductory physics course, mainly taken by engineering, chemistry, mathematics, and physics majors [60]. Approximately one-fifth of the students in these courses are females. This course covers electricity and magnetism and some wave optics, and calculus is used extensively in the course since students are supposed to have taken calculus before they take this course. It is taken after the first introductory physics course, which covers mechanics and waves. Most of the several hundred students in different sections of this course were college freshmen. The students in this course had $4 \mathrm{~h}$ of lecture time and $1 \mathrm{~h}$ of recitation time. The different sections of the course were generally taught by different instructors and the recitations were taught by graduate teaching assistants. All of the sections of the course discussed in this investigation primarily had traditional lecture-based instruction in the $4 \mathrm{~h}$ of lecture time, and in the recitations, the graduate teaching assistants fielded questions about the homework from the students and solved example problems on the board (except in the sections of the course that we designate the experimental group, in which students worked on the tutorial in recitation class after lecture-based instruction in those concepts).

The reason there are several sections of this same course offered in the same semester at the university where the investigation was carried out (University of Pittsburgh) is that this course is mandatory for several hundred engineering freshmen and also for other majors, e.g., from chemistry, mathematics, etc. The content covered by all the different sections of the course (both experimental and comparison groups) is the same. Each week students were asked to do homework from the textbook (introductory physics textbook by Halliday, Resnick, and Walker) except that in the experimental group, they were asked to complete as homework the part of the tutorial that students did not complete in class, in addition to the textbook homework. In all sections of the course, each week after students submitted the homework on a particular topic, they were given a quiz on that material in the last 15-20 min of the recitation class.

We note that this investigation employs a quasiexperimental design [60] in that we did not have control over whether a particular student will be in the section of the course in which the tutorial was used and we did not have control over who their instructor and teaching assistant (for the recitations) would be. Also, although students in all sections of the course used the same textbook, we did not have control over the textbook homework assignments or the midterm and final exam given by the instructors of different sections (since the instructor of each section in the same semester had full control of their section of the course). However, the conceptual survey of electricity and magnetism [52] given to some of the sections in the previous years as a pretest and post-test suggests that student performance on average in various sections of the course is comparable at the beginning of the course (pretest) and on the post-test after traditional lecture-based 
instruction. We note however that this is not a randomized experimental design and one should keep that in mind in interpreting the results.

The development of the research-validated guided inquiry-based tutorial was carried out with the following core issues in mind: (i) the tutorial must build on students' prior knowledge so it is important to investigate the difficulties students have related to relevant concepts before the development of the tutorial; (ii) the tutorial must create an active learning environment where students get an opportunity to build a good knowledge structure in which there is less room for misconceptions; (iii) the tutorial must provide scaffolding support, guidance, and feedback to students and opportunity to organize, reconstruct, and extend their knowledge.

The process of the development and validation of the questionnaire and tutorial spanned three years and started with a cognitive task analysis [61-62] from the perspective of an expert and an investigation of the common difficulties that introductory physics students in this course have with the electric field and superposition principle, especially in the case of continuous charge distribution. Thus, the preliminary version of the questionnaire (which was refined into pretest and post-test questions) and the tutorial not only used research on student difficulties as a guide but also a cognitive task analysis of the underlying concepts from an expert perspective. The cognitive task analysis from the perspective of an expert involves making a fine-grained flow chart of the concepts involved in solving a specific class of problems [61-62]. Such analysis can help identify some stumbling blocks where students may have difficulty. However, investigation of students' difficulties using written tests and interviews was critical for developing the tutorial because theoretical analysis from the perspective of an expert often does not capture all of the difficulties students have with relevant concepts.
Table I summarizes this process for data collection before, during, and after the development and validation of the questionnaire and different versions of the tutorial. The student difficulties were investigated by administering validated open-ended and multiple-choice questions in written form to introductory students in various sections of the course after traditional instruction in relevant concepts and via individual interviews with a subset of students. These open-ended and multiple-choice questions were validated with the help of physics instructors who had taught this course several times (the questions were iterated with them to ensure that they were robust and interpreted unambiguously by experts) and introductory physics students to ensure, e.g., that they interpreted the questions as intended. Since the validated versions of the open-ended questions became the pretest and post-test questions, we will focus on student difficulties vis-à-vis their performance on the pretest and post-test questions. The validation of the multiple-choice questions is discussed elsewhere [58]. Individual interviews were conducted using a semistructured, think-aloud protocol to better understand the rationale for student responses before, during, and after the development of different versions of the tutorial and the corresponding pretest and post-test. During the semistructured interviews, introductory students were asked to verbalize their thought processes while they answered the questions. Students read the questions related to the electric field and superposition principle including in the case of continuous charge distribution and answered them to the best of their ability without being disturbed. We prompted them to think aloud if they were quiet for a long time. After students had finished answering a particular question to the best of their ability, we asked them to further clarify and elaborate issues that they had not clearly addressed earlier.

The guided inquiry-based tutorial is conceptual in nature. The tutorial was implemented in a manner in which

TABLE I. Procedure for data collection before, during, and after the development and validation of the questionnaire and different versions of the tutorial.

Before the development of the questionnaire and tutorial

- Cognitive task analysis from an expert perspective of the concepts underlying electric field due to continuous charge distribution

- Development of questions and using them for discussions with instructors teaching the course and iterating the questions with the instructors to validate them

- Several years of examining student responses to

- open-ended questions

- multiple-choice questions

- Individual interviews with introductory physics students $(N=5)$
During and after the development of the preliminary version of the tutorial: Individual interviews with introductory physics students and individual discussions with introductory physics course instructors

- One-on-one interviews with introductory physics students $(N=10)$ examining responses to

- tutorial pretest questions

- inquiry-based learning sequences in tutorial

- tutorial post-test questions

- Discussions with instructors

- Refinement of the tutorial and corresponding pretest and post-test based upon feedback from students and instructors 
students worked on them in small groups in their recitation. However, whatever part of the tutorial students could not finish in the recitation, they were asked to finish as part of their homework. During the development of the tutorial, in the individual interviews, we administered the pretest, tutorial, and post-test to some introductory physics students, who were asked to think aloud while working on them. After each administration, we modified the tutorial based upon the feedback obtained from student interviews. The tutorial was also iterated several times with four physics instructors who had taught the introductory electricity and magnetism course for their feedback, and modified after each round of feedback. These individual administrations helped fine-tune the tutorial and improve its organization and flow. In summary, the development of the tutorial went through a cyclic, iterative process which included the following stages before the in-class implementation in several sections of the introductory physics course: (i) Development of the preliminary version based on a cognitive task analysis from an expert perspective of the underlying knowledge and research on student difficulties; (ii) implementation and evaluation of the tutorial by administering it individually to students and obtaining feedback from instructors who teach the relevant introductory course; (iii) determining its impact on student learning and assessing what difficulties were not adequately addressed by the tutorial; (iv) refinements and modifications based on the feedback from the implementation and evaluation.

Table II is a summary of activities in the experimental group (tutorial group) and comparison group (nontutorial group) in different sections of the course in the semester in which the final version of the tutorial (along with the pretest and post-test) was administered in class in some sections of the course. For all the different sections in Table II, $N_{1 \text { pre }}$, $N_{2 \text { pre }}, N_{3 \text { pre }}$, and $N_{4 \text { pre }}$ refer to the number of students who took the pretest and $N_{1 \text { post }}, N_{2 \text { post }}, N_{3 \text { post }}$, and $N_{4 \text { post }}$ refer to the number of students in each section who took the post-test (only matched students who took both the pretest and posttest are included when students took both the pretest and post-test as in $N_{2}, N_{4}$ ). In Table II, the timeline or chronological order is from left to right. As can be seen from Table II, both groups had traditional lecture-based instruction in relevant concepts. After the traditional lecture-based instruction, students in some of the classes (regardless of whether they were in the experimental or comparison group) took the pretest in class as a quiz which was graded for completeness (all students obtained the full quiz score for trying their best). Also, the pretest was neither returned to the students nor was it discussed by the instructors with the students. Then all students worked on the textbook homework problems. After submitting the homework on relevant concepts, students in the experimental group worked on the tutorial in small groups in recitation class. The recitation classes in which students worked on the tutorial were run by teaching assistants (TAs) who were provided training in how to run a tutorial session effectively. Typically, students worked in small groups and they were asked to raise their hands for questions and clarifications. The comparison group (nontutorial group) only had traditional textbook homework. After students in the comparison group submitted their homework on these concepts in the recitation class, they

TABLE II. Summary of activities in the experimental group (tutorial group) and comparison group (nontutorial group) in different sections of the introductory physics course. For all the different sections in Table II, $N_{1 \text { pre }}, N_{2 \text { pre }}, N_{3 \text { pre }}$, and $N_{4 \text { pre }}$ refer to the number of students who took the pretest and $N_{1 \text { post }}, N_{2 \text { post }}, N_{3 \text { post }}$, and $N_{4 \text { post }}$ refers to number of students in each section who took the post-test (only matched students who took both the pretest and post-test are included when students took both the pretest and post-test as in $N_{2}, N_{4}$ ).

\begin{tabular}{|c|c|c|c|c|}
\hline & & $\begin{array}{l}\text { Pretest (quiz graded } \\
\text { for completeness) }\end{array}$ & $\begin{array}{l}\text { Learning activities after lecture-based } \\
\text { instruction and pretest }\end{array}$ & $\begin{array}{l}\text { Post-test (quiz } \\
\text { graded for } \\
\text { correctness) }\end{array}$ \\
\hline $\begin{array}{l}\text { Tutorial group or } \\
\text { experimental } \\
\text { group (worked } \\
\text { through the tutorial } \\
\text { after traditional } \\
\text { instruction) }\end{array}$ & $\begin{array}{l}\text { Traditional lecture-based } \\
\text { instruction in relevant } \\
\text { concepts for both } \\
\text { groups (total time } \\
\text { same for both groups } \\
\text { but less time spent by } \\
\text { instructor solving }\end{array}$ & $\begin{array}{c}N_{1 \text { pre }}=0 \\
N_{2 \text { pre }}=64 \\
N_{3 \text { pre }}=0\end{array}$ & $\begin{array}{l}\text { Worked on textbook homework } \\
\text { problems on relevant concepts. } \\
\text { Worked on tutorial in recitation } \\
\text { class in small groups before taking } \\
\text { the post-test. Students had } \\
\text { opportunity to ask questions. }\end{array}$ & $\begin{array}{l}N_{1 \text { post }}=87 \\
N_{2 \text { post }}=64 \\
N_{3 \text { post }}=64\end{array}$ \\
\hline $\begin{array}{l}\text { Nontutorial group or } \\
\text { comparison group } \\
\text { (did not work } \\
\text { through the } \\
\text { tutorial) }\end{array}$ & $\begin{array}{l}\text { example problems } \\
\text { during lecture in the } \\
\text { tutorial group since } \\
\text { students in that group } \\
\text { worked on tutorial } \\
\text { during part of class) }\end{array}$ & $N_{4 \text { pre }}=57$ & $\begin{array}{l}\text { Worked on textbook homework } \\
\text { problems on relevant concepts. } \\
\text { Students had opportunity to ask } \\
\text { questions about relevant concepts } \\
\text { (including the textbook } \\
\text { homework). TA solved example } \\
\text { problems from textbook before } \\
\text { administering post-test. }\end{array}$ & $N_{4 \text { post }}=57$ \\
\hline
\end{tabular}


had the opportunity to ask questions about any doubts they had and then the teaching assistant (TA) worked out some example problems from the textbook before giving the posttest as a quiz to students. The post-test quiz was graded for correctness in all sections. The weight assigned to the posttest was the same as the pretest and each counted for less than $1 \%$ of the course grade. To summarize, in the tutorial group, as shown in Table II, the number of matched students who were administered both pretest and post-test ranged from 57 to 64 (since two of the sections as shown in Table II did not take the pretest due to the time constraints). We note that although the pretest and post-test accompanying the tutorial assess the same concepts, the same test was not used to minimize the effect of the pretest on the post-test. While the pretest was not returned, the post-test was returned after grading.

\section{STUDENT DIFFICULTIES}

As summarized in Table I, student difficulties were investigated by developing and validating questions and administering them in the relevant introductory physics course in written format as well as by interviewing a subset of students using think-aloud protocol to better probe their reasoning. After the validation process, many of the probing questions were transformed into the pretest and post-test questions. Therefore, here we will restrict ourselves mostly to student difficulties displayed in response to the pretest and post-test questions either in written responses or in individual interviews. However, before we discuss student difficulties found, we note that students who used the tutorial as a pedagogical tool were less likely to have the difficulties after working on the tutorial (on the post-test) than on the pretest (after traditional lecture-based instruction only). For reference, the entire pretest and post-test for the tutorial are included in the Appendix. Also, the difficulties on the pretest and post-test were similar in nature for both the tutorial group and an equivalent comparison group consisting of students who did not work on the tutorial (and only learned this material via traditional lecture-based instruction). The main difference between these groups was that the tutorial group students were significantly less likely to have the difficulties after working on the tutorial (as discussed later in the tutorial assessment section).

All questions on the pretest and post-tests require the use of the superposition principle to find the electric field at various points for a given continuous charge distribution. Many students had great difficulty with exploiting the principle of superposition to obtain the net field at a point due to the continuous charge distribution present in the region. In other words, the performance of many students on the pretest and post-tests was closely tied with their understanding of the principle of superposition pertaining to the electric field and the ability to apply it to the case of a continuous charge distribution. Below, we first discuss data on the common student difficulties found without separating the performance of the tutorial group and comparison (nontutorial) group on the pretest and post-tests. In a later section, we will present quantitative data comparing the performances of the tutorial and comparison groups and how students who worked on the tutorial performed on these concepts on the post-test compared to those in the nontutorial groups.

Difficulties in generalizing from discrete to continuous charge distribution.-We find that the difficulty in using Coulomb's law and the principle of linear superposition to find the net electric field at a point was, in general, exacerbated due to the fact that the charge distribution was continuous. Interviews suggest that sometimes a student who knew how to calculate the net electric field at a point, e.g., due to an electric dipole, by drawing the electric field due to individual charges and then finding their vector sum did not know how to generalize that conceptual and procedural knowledge for a charge distribution which was continuous. In particular, in the interviews, when some students struggled with the questions with the continuous charge distribution, they were asked to find the electric field due to an electric dipole. They sometimes correctly laid out a procedure for calculating the electric field in the dipole situation based upon what they had done in class or in homework but they had difficulty in extending those concepts and procedures they used in the dipole case to situations with continuous charge distributions (and sometimes for questions with several discrete charges) even when explicitly encouraged to do so. For example, the pretest questions (1) and (2) focus on a continuous charge distribution. The underlying reasoning for approaching these questions is analogous to the discrete case in that students still need to use Coulomb's law and the superposition principle (although it is significantly more complex due to the continuous nature of the charge distribution). In particular, in Fig. 1, since all of the positive charges on finite uniform line of charge are below and to the right side of $A$ and $A^{\prime}$, the electric field at $A$ and $A^{\prime}$ should have an upward component and a component to the left (so that the net electric field at these points is at an angle less than $90^{\circ}$ above left). Similarly, for points $C$ and $C^{\prime}$, the electric field should point above right at some angle less than $90^{\circ}$. Since half of the charge is on the left and half is on the right of points $B$ and $B^{\prime}$ and the charges are symmetrically situated with respect to those points, the horizontal components of the field cancel out and the net field at points $B$ and $B^{\prime}$ is vertically upward. Thus, the direction of net electric field at points $B$ and $B^{\prime}$ is the same. However, since point $A^{\prime}$ is closer to the charges, the horizontal component of the net field at point $A^{\prime}$ is larger than that at point $A$. Therefore, the net field at point $A^{\prime}$ makes a smaller angle with the horizontal direction (left) than that at point $A$.

The distribution of student responses to this question are summarized in Table III and shows that many students correctly found the direction of the net electric field at points $B$ and $B^{\prime}$. Also, out of 132 introductory physics students who answered the question about the direction of the field at points $A$ and $C, 67 \%$ provided the correct 
(1) Consider the three points A, B and C shown below, each at a distance $L$ above a finite uniform line of positive charge of length $2 L$. Draw arrows to show the direction of the net electric field at each of these three points. Explain.

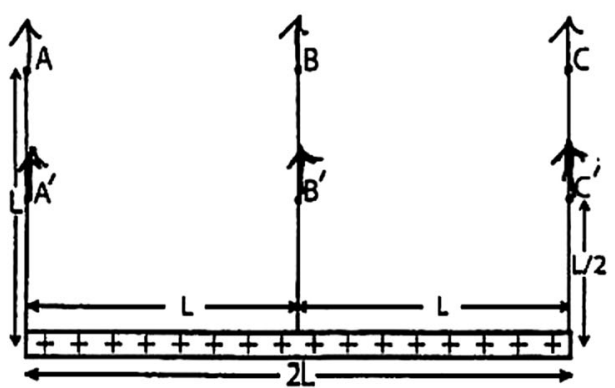

FIG. 1. A sample response from a student for pretest questions (1) and (2) for the direction of the field at the points shown.

answer. The most common incorrect answer was that the direction for all points is upward.

One interviewed student who found the direction of the electric field correctly for the electric dipole said that he never understood how to handle continuous charge distributions and found those problems difficult. He did not appropriately apply the principle of linear superposition to the continuous charge distribution cases posed and attempted to use memorized knowledge for the case of the infinite uniform line of charge to guess the answers. In particular, for the uniformly charged finite rod, he claimed that the electric field is outward and perpendicular to the rod even though the rod is finite in length. Other students had similar difficulties. A typical response with this type of difficulty on the pretest questions (1) and (2) is shown in Fig. 1.

Interviews suggest that many students found the continuous charge distribution problems to be very challenging and they did not have a functional understanding of the fact that the same superposition principle that is used for discrete charges can be systematically extended for a continuous charge distribution even though the charge distribution is significantly more complex. Interviews also suggest that some of the students had not thought carefully about how to calculate the electric field at a point due to a continuous charge distribution or the symmetry considerations that are useful for making qualitative predictions about the net electric field due to the continuous charge distributions in the questions posed.

TABLE III. Distribution of student responses for the directions of electric field at various points on pretest question (1) including all sections that took the pretest (after traditional lecture-based instruction) in relevant concepts. The percentages of students who answered the questions correctly are in boldface and underlined.

Direction of field Direction of field Direction of field at points $A$ or $A^{\prime}$ at points $B$ or $B^{\prime}$ at points $C$ or $C^{\prime}$

\begin{tabular}{lccc}
\hline$\uparrow$ & $24 \%$ & $\underline{95 \%}$ & $24 \%$ \\
$\nwarrow$ & $\underline{\mathbf{6 7 \%}}$ & 0 & $3 \%$ \\
$\nearrow$ & $3 \%$ & 0 & $\mathbf{6 7 \%}$ \\
Others & $6 \%$ & $5 \%$ & $6 \%$ \\
\hline \hline
\end{tabular}

Incorrectly claiming that electric field is the same at points which do not have the same symmetry with respect to the charge distribution.-As noted, the principle of superposition was extremely challenging for many students in the context of a continuous charge distribution. Many students struggled to reason about the electric field conceptually by exploiting the symmetry of the charge distribution given in the questions posed. For example, on the post-test question (4), students were given a finite sheet with charge uniformly distributed on the surface and were specifically given two points equidistant from the sheet (see the Appendix). One of the points is above the center of the sheet and the other is close to a corner. Students were asked to compare the magnitude and direction of the electric field at these two points. A similar question was posed immediately after that question in which the finite sheet with uniform charge was replaced with an infinite sheet with uniform charge in order to investigate whether students can discern the difference between them. Many students, especially those in the nontutorial group, did not recognize the difference between an infinite and finite sheet with uniform charge and claimed that the electric fields have the same magnitude and direction in both of these situations since the charge distribution is uniform.

In particular, on the post-test question (4) (see Fig. 2), students were given a square sheet of length $L$ on each side on which positive charge is uniformly distributed with a charge per unit area (surface charge density). There are two points given, each at a height $h=L / 2$ above the sheet: point $C$ is directly above the center of the sheet and point $B$ is off center (see the Appendix). Students were asked to compare the electric field at points $B$ and $C$. In fact, a similar question was asked to 541 introductory students in the multiple-choice format and some students incorrectly claimed that the electric field at points $B$ and $C$ have the same magnitude and same direction (20\%), same magnitude but different directions $(15 \%)$, and different magnitudes but same direction (7\%) [58]. Those who incorrectly claimed that the magnitude of the electric field is the same at both points but not the direction often justified it by citing that the vertical distance of points $B$ and $C$ from the finite uniform sheet of charge is the same so the magnitudes are the same but the direction of the electric 


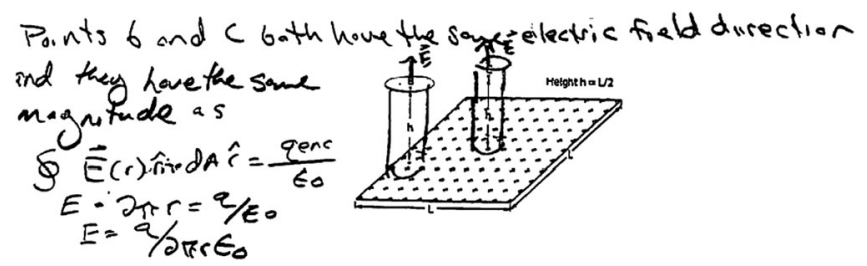

FIG. 2. A sample response from a student for the post-test question (4).

field at point $C$ above the center is perpendicular to the sheet but not at point $B$ near the sheet edge. Those who stated that the direction of the electric field at points $A$ and $B$ are the same often incorrectly claimed that the direction of the electric field is outward from the sheet perpendicular to the sheet at both points. Students who claimed that both the magnitudes and directions of the electric field must be the same at both points incorrectly claimed that this was true because the sheet has a uniform charge and points $B$ and $C$ are at the same heights above the sheet. Figure 2 shows a typical reasoning of a student who thought that both the magnitude and direction of the electric field must be the same at both points.

Discerning the symmetry of the charge distributions was difficult for many students in other questions as well. Interviews suggest that some students had not understood the symmetry considerations involved in reasoning that the magnitude of the electric field due to an infinite uniform line of charge is constant at a fixed perpendicular distance from it (and straight out perpendicular to the line of charge) and the magnitude of the electric field due to an infinite uniformly charged thin sheet is constant everywhere (but opposite in direction on the two sides of the sheet). As noted, they often claimed that the results for the finite uniform line of charge or finite uniform sheet of charge should be the same, at least for the direction of the electric field as in the corresponding infinite case, not recognizing the difference between a finite and infinite line or sheet of charge. Table III shows that about $24 \%$ of the students thought that the direction of the electric field at the points which are the same distance away from a finite uniform line of charge is the same. Interviews with some of these students suggest that they were confused due to the fact that the electric field at all points that are the same distance away from an infinite uniform line of charge is the same.

In summary, interviews suggest that with only traditional lecture-based instruction, some students could only recall memorized results for the electric field for highly symmetric cases (e.g., infinite line or sheet with uniform charge or a sphere with uniform surface charge) and had not thought deeply about the conditions under which these results for the field are valid. Some of the interviewed students appeared to be quite confident that the finite line or finite sheet with uniform charge will have the same electric field at points which are the same perpendicular distance away from the line or sheet because the charge on them is "uniformly spread out." Even explicitly pointing out to students during interviews that the line or sheet on which the charge is uniformly distributed was finite generally did not help. In the interviews, asking students who provided these types of responses to explicitly work out how they would calculate the electric field at different points from the line or sheet with uniform charge that had different symmetries was often not productive. Students with these responses often failed to recognize that the magnitude of the electric field at any point above an infinite sheet or line with uniform charge at a fixed distance away is the same. However, this result only applies in the limiting case when the sheet or the line with uniform charge is infinite.

The electric field is radially out without taking into account the symmetry of the charge distribution.-After traditional lecture-based instruction, some students claimed that the electric field must be radially out from the point where they were asked to find the field (somewhat similar to the electric field due to a point charge) and did not take into account the symmetry of the charge distribution causing the electric field at those points. For example, the pretest questions (1) and (2) are about a finite uniform line of positive charge of length $2 L$ (see the Appendix). Points $A, B$, and $C$ each are at a distance $L$ above the line of charge and points $A^{\prime}, B^{\prime}$, and $C^{\prime}$ are at a distance $L / 2$ above the line of charge. Students are asked to draw arrows to show the approximate direction of the net electric field at each of these six points and to compare how the direction of the net field at point $A$ compares with $A^{\prime}$ and the direction of the field at point $B$ compares with $B^{\prime}$. In response, some students drew diagrams similar to that shown in Fig. 3. Upon questioning about why the net electric field at a point is pointing in so many different directions, one student who drew such a diagram stated that since "the electric field due to point charges at points $A, B$, and $C$ will point in all directions so the electric field cannot be directed perpendicular to the finite line of charge everywhere." Further discussions suggest that some of the introductory students with this type of response were confused about the electric field due to each infinitesimal charge that makes up

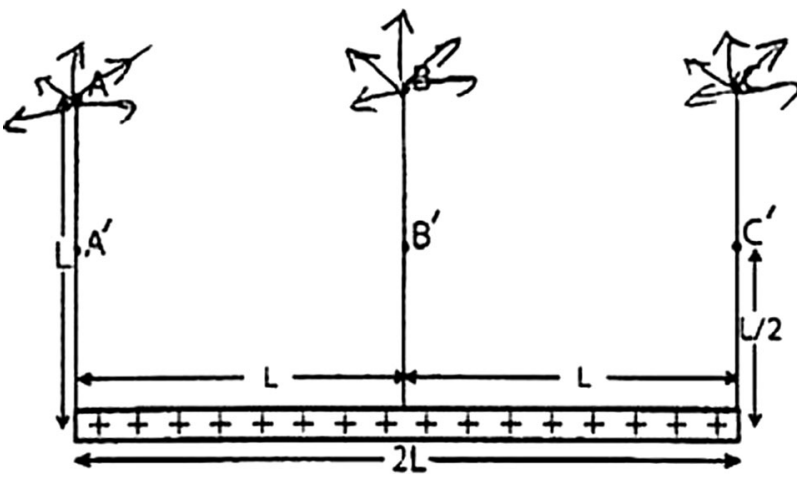

FIG. 3. A sample drawing from a student for pretest question (1) showing the direction of the electric field at points $A, B$, and $C$. 
the line of charge vs the net electric field. Some of the confusion was also due to the inability to make a clear distinction between the electric field due to a point charge being radially outward vs the electric field at a point due to a charge distribution having a well-defined direction.

Difficulty in visualizing in three dimensions. - Interviews suggest that visualizing in three dimensions is often challenging for introductory physics students. For example, on pretest question (4) (see the Appendix), students were asked to draw all of the points that have the same magnitude of electric field as a point shown close to an infinite uniformly charged cylinder. In a cross-sectional view, all such points form a circle, and in a side view, all such points form an infinite cylinder passing through the point given. We find that it was easier for many students to provide the correct response in the two-dimensional cross-sectional view, but often due to incorrect reasoning. For example, due to the difficulty in visualizing the situation in three dimensions, some of the students claimed that if the cross-sectional view is a circle, the three-dimensional surface formed by all such points should be a sphere. Some interviewed students explicitly emphasized that in a three-dimensional view, the surface should be a sphere even though they had drawn the surface as a circle in the two-dimensional view. Interviews also suggest that some students had less difficulty in answering the question in the two-dimensional cross-sectional view because both a cylinder and a sphere would be represented as a circle (the points they were asked for in the pretest question 4 will form a circle). However, they often struggled to visualize the situation in three dimensions correctly and realize that the circle was the cross-sectional view of an infinitely long cylinder in the situation provided.

Difficulty in setting up an appropriate integral for a finite line of uniform charge.-On all of the pretest and posttest questions, students were not asked to perform any challenging integrals to find an expression for the electric field due to the continuous charge distribution. They were only asked to draw the arrows showing the electric field qualitatively for the continuous charge distributions or predict whether the magnitude of the electric field will be the same at two different points due to a given charge distribution based upon symmetry considerations. Since students struggled to use symmetry considerations conceptually to infer the features of the electric field, in some interviews, we asked students to set up an integral for finding the field magnitude due to a finite line with uniform charge at the perpendicular bisector at a certain distance from the line of charge. Students had difficulty using Coulomb's law and invoking the principle of linear superposition and vectorially adding the field due to the infinitesimal point charges that make up the continuous charge distribution to find the field due to the continuous charge distribution. Even students who were able to find the field at a point due to discrete point charges had great difficulty with how to apply the superposition principle to a continuous charge distribution and how one can break up the continuous charge distribution into infinitesimal elements of charge (length, area or volume) to find the net field at a point by vectorially adding (or integrating over) the contributions to the field due to the entire charge distribution. Also, during individual interviews, two students were confused about the fact that for the continuous charge distribution, one is supposed to consider differential charge $d \mathbf{q}$. They did not realize that $d \mathbf{q}$ simply refers to a very small amount of charge and wondered why it is valid to consider charge $d \mathbf{q}$ (which they thought would be less than the charge on a proton or electron) when charge is supposed to be indivisible.

\section{AN OVERVIEW OF THE TUTORIAL}

The guided inquiry-based tutorial, which can be found at Ref. [63], uses student difficulties found via research as resources and strives to help students learn how to use Coulomb's law and the superposition principle in the context of a continuous charge distribution. It strives to help students learn how to reason about problems which involve continuous charge distribution by leveraging what they have already learned in the case of a discrete charge distribution using Coulomb's law and superposition principle. Since generalizing from discrete to continuous charge distribution is one common difficulty found via research, the tutorial starts with questions that ask students to predict the electric field at a point due to five collinear equidistant identical positive point charges shown in Fig. 4(a). The students are told that the direction of the electric field at point $A$ due to only the three middle charges in Fig. 4(a) is shown by the arrow labeled $\vec{E}_{234}$. The coordinate axes shown in Fig. 4(b) is introduced so that students can provide the answers for the guided-inquiry questions posed to them with respect to the coordinate axes and students are asked to reflect upon why these coordinate axes may be convenient for comparing the electric field in different

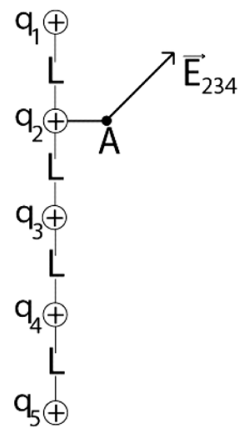

(a)

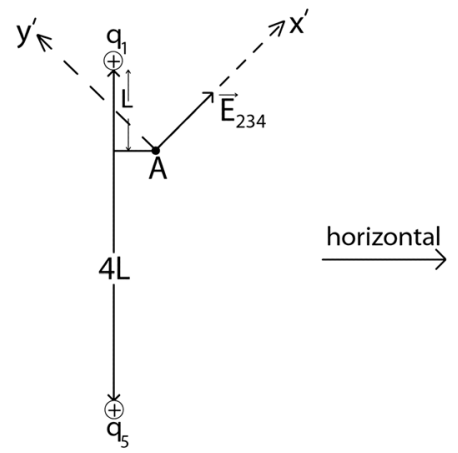

(b)
FIG. 4. A schematic diagram from the tutorial designed to help students learn to transition from the case of the electric field due to a discrete distribution of equidistant identical charges in a straight line to the field due to a continuous charge distribution. 
situations posed to them as part of the guided learning sequence. The students are then asked to draw the direction of the electric field at point $A$ only due to identical charges $q_{1}$ and $q_{5}$. Then they are asked to find the components of the electric field along the $x^{\prime}$ and $y^{\prime}$ directions due to all five charges so that they can compare the components of the net electric field due to all five charges along these directions with that of $\vec{E}_{234}$. Based on the comparison, students are guided to infer whether the net electric field at point $A$ due to all of the five identical charges makes a larger or smaller angle with the horizontal than $\vec{E}_{234}$. The number of collinear identical point charges are gradually increased by adding two more identical charges at each end and asking students to predict how the electric field at point $A$ will be impacted, e.g., by having seven collinear identical charges as opposed to the initial five. In particular, the students are asked to consider how the angle with the horizontal at point $A$ changes when two more identical charges $q_{0}$ and $q_{6}$ are symmetrically added at the two ends to extend the five charges in a row to seven identical equispaced charges in a row. Then, the guided inquirybased sequence provides further scaffolding support and asks students to extend this charge distribution by adding more identical collinear charges at the two ends and predict the direction of the electric field at point $A$ in the limit of a very large number of identical positive charges in a straight line separated by equal distance $L$.

Next, the guided inquiry-based sequences in the tutorial ask students to consider the case when the charge is uniformly distributed on an infinite line and strive to help students recognize the features of a uniform continuous line of charge. Students are asked to predict the direction of the net electric field at point $A$ for the uniform continuous infinite line of charge and compare it with the earlier discrete cases.

As noted earlier, other common difficulties found via research include incorrectly claiming that the electric field is the same at points which do not have the same symmetry with respect to the charge distribution and claiming that the electric field is radially out without taking into account the symmetry of the charge distribution. To reduce these types of difficulties, students are then guided through tasks in which they have to contemplate the symmetry of the charge distribution. For example, they are asked to find the points that have the same net electric field (same magnitude and same direction) as that at point $A$ due to the infinite line with uniform charge. They are also asked to find the points at which the magnitude of the net electric field is the same as that at point $A$ in Fig. 4 (but not necessarily the same direction). Moreover, since one of the common difficulties found via research was the challenge associated with visualizing in three dimensions, the students are guided to draw a sketch showing the points satisfying these requirements in a side view and a cross-sectional view to help them learn to visualize the field in three dimensions. Based on the sketches, the students are guided to predict the shape of the three-dimensional surface on which the magnitude of the net electric field is the same everywhere, but the direction may be different due to an infinite uniform line of charge.

Since recognizing the symmetry of the charge distribution was particularly challenging for students as discussed earlier, after engaging students with the symmetry properties of a uniformly distributed infinite line of charge, students are guided through the case of an infinitely long hollow cylinder with uniform surface charge. The guided inquiry-based approach helps students recognize that an infinitely long uniform line of charge has the same type of symmetry properties as an infinitely long uniform cylinder of charge. Later in the tutorial, students are asked to reflect upon the validity of statements by different hypothetical students (some of whom are correct and some incorrect) which strives to provide further scaffolding support to help students recognize that the symmetry properties of a finite line and an infinite line with uniform charge are different and only a few symmetries of the charge distribution are sufficiently high that one can predict the direction of the electric field everywhere easily. These types of reflection tasks that explicitly require comparison between finite and infinite uniform line of charge are designed based upon the common difficulties discussed earlier with the symmetry of the charge distribution and recognizing when the electric field is radial from the line of charge and when it is not.

\section{PERFORMANCE OF THE TUTORIAL AND COMPARISON GROUPS}

The pretest and post-test (see the Appendix) were graded by two researchers based upon an agreed rubric, and the interrater reliability was better than $85 \%$. The grading rubric scores each answer as correct or incorrect (based upon whether the student responses were conceptually correct or not) and if there was an explanation required, student responses for that part of the question were scored on a three-level scale (full point for correct explanation, zero point for incorrect or no explanation and half point for partially correct). Below we discuss an example of a question given on the post-test and how the students in the tutorial and nontutorial groups performed on it before comparing the average scores of the tutorial and nontutorial groups on each question.

On post-test question (4) (see the Appendix), students are asked to show the direction or approximate direction of the electric field at each point and compare the magnitudes of the net field at the two points. This question is similar to the one given in the pretest [questions (1) and (2)] except that the pretest questions are about the uniformly charged finite line instead of a finite sheet of charge. To answer this question correctly, the students must recognize that the sheet is finite. Point $C$ is right above the center of the sheet, so the electric field at point $C$ is perpendicular to the sheet. However, point $B$ is closer to one corner of the sheet, so the electric 
TABLE IV. Distribution of the student responses to post-test question (4) for the tutorial and nontutorial groups. The percentages of students who answered the questions correctly are in boldface and underlined.

\begin{tabular}{|c|c|c|}
\hline & $\begin{array}{l}\text { Tutorial } \\
\text { group }\end{array}$ & $\begin{array}{l}\text { Nontutorial } \\
\text { group }\end{array}$ \\
\hline \multicolumn{3}{|l|}{ Direction of the field at point $B$} \\
\hline perpendicular to the sheet & $13 \%$ & $15 \%$ \\
\hline not perpendicular & $\underline{82 \%}$ & $46 \%$ \\
\hline no answer or other answers & $\overline{5 \%}$ & $\overline{40 \%}$ \\
\hline \multicolumn{3}{|l|}{ Direction of the field at point $C$} \\
\hline perpendicular to the sheet & $95 \%$ & $62 \%$ \\
\hline not perpendicular & $\overline{0.5 \%}$ & $\overline{2 \%}$ \\
\hline no answer or other answers & $4.5 \%$ & $36 \%$ \\
\hline \multicolumn{3}{|c|}{ Magnitudes of the field at points $B$ and $C$} \\
\hline$E_{B} \neq E_{C}$ or $E_{B}<E_{C}$ & $\underline{75 \%}$ & $34 \%$ \\
\hline$E_{B}=E_{C}$ & $19 \%$ & $32 \%$ \\
\hline$E_{B}>E_{C}$ & $1.5 \%$ & $2 \%$ \\
\hline no answer or other answers & $4.5 \%$ & $32 \%$ \\
\hline
\end{tabular}

field at point $B$ is not perpendicular to the sheet. Also, the magnitudes of electric field at points $B$ and $C$ are not equal.

Table IV shows the distributions of student responses from the tutorial group and nontutorial group using the correct or incorrect part of the rubric (scores shown in Tables V-VIII were obtained including the reasoning part of the rubric as well). The table shows that $95 \%$ of the students from the tutorial group provided the direction of the electric field at point $C$ correctly and $82 \%$ of them provided the direction of the field at point $B$ correctly. Moreover, $75 \%$ of these students in the tutorial group compared the magnitudes of the field at $B$ and $C$ correctly. Table IV shows that in the nontutorial group, the percentage of students providing the correct response is lower and about one-third of the students thought that the magnitude of the electric field at both points should be the same. Moreover, $30 \%-40 \%$ of the students in the nontutorial group provided no answer or other answers to these posttest questions compared to roughly $5 \%$ in the tutorial group that suggests that the tutorial was effective in helping students with these concepts. We note that this question was not asked on the pretest but the average score was less than $40 \%$ on the pretest (see Tables V and VI) for both the tutorial and nontutorial groups and a large number of students in both groups were equally confused.

Table V shows the average pretest and post-test scores for three sections of the course in which students engaged with the tutorial. The pretest was only administered in Sec. II in the tutorial group due to time constraints in other sections and instructors' willingness. Table $\mathrm{V}$ shows that the average performance in Sec. II was significantly better on the posttest compared to the pretest. Although all three sections of the course performed significantly better on the post-test compared to the pretest performance of Sec. II, Sec. II performed
TABLE V. Average percentage total scores on the pretest and post-test for the tutorial group. The pretest was administered after traditional instruction but before the tutorial only in Sec. II due to time constraints in other sections. The symbol $N$ refers to the matched number of students in a given section who took both the pretest and post-tests.

\begin{tabular}{lccc}
\hline \hline Section & $N$ & Pretotal & Post-total \\
\hline 1 & 87 & $\ldots$ & $79 \%$ \\
2 & 64 & $38 \%$ & $92 \%$ \\
3 & 64 & $\ldots$ & $77 \%$ \\
\hline \hline
\end{tabular}

better than Secs. I and III. The differences in the performance of different sections of the tutorial group on the post-test may be due to the differences in student samples, instructor or TA differences, or the manner in which the tutorial was incentivized and administered.

Table VI shows the pretest and post-test data from a comparison group which consists of a section in which the students did not engage with the tutorial. As noted, the total class time devoted to these topics was equivalent in this class to the time spent by the instructors in the tutorial groups. The pretest was given to the comparison group immediately after relevant instruction, similar to the tutorial group. The post-test was given the following week as part of the weekly recitation quizzes after students had the opportunity to complete all the homework problems related to those topics.

The results of a $t$ test that compares the performance of the tutorial and nontutorial groups on the pretest and post-tests in Tables V and VI show that regardless of which group the students belong to (i.e., whether they belonged to the tutorial or comparison groups), their performance on the pretest was poor after traditional instruction (the averages for tutorial and nontutorial classes are not statistically significantly different with a $p$ value of 0.945 ). On the other hand, students in the comparison group did significantly worse on the post-test than the students in the tutorial group ( $p$ value $<0.0001$ ). It is worth noting that the post-test performance of all three sections of the course in which students engaged with the tutorial is significantly better than the performance of students in the nontutorial group.

TABLE VI. Average percentage total scores on the pretest and post-test for the section taught using traditional lecture only (nontutorial or comparison group). The pretest was administered after lecture-based instruction in class. The post-test was administered in recitation the following week after students submitted their homework on these topics and had the opportunity to ask questions about any doubts they had and the TA solved some textbook problems on the blackboard on relevant concepts.

\begin{tabular}{lcc}
\hline \hline$N$ & Pretotal & Post-total \\
\hline 57 & $38 \%$ & $55 \%$ \\
\hline \hline
\end{tabular}


TABLE VII. Percentage average total pretest and post-test scores (matched pairs) divided into three groups according to the pretest performance for the tutorial group. $N_{2}$ denotes the total number of students in Sec. II who worked through the tutorial and took both the pretest and post-tests.

\begin{tabular}{lccc}
\hline \hline Pretest range (\%) & $N_{2}$ & Pre & Post \\
\hline All & 64 & $38 \%$ & $92 \%$ \\
$0 \%-34 \%$ & 28 & $19 \%$ & $88 \%$ \\
$34 \%-67 \%$ & 34 & $51 \%$ & $95 \%$ \\
$67 \%-100 \%$ & 2 & $85 \%$ & $95 \%$ \\
\hline \hline
\end{tabular}

TABLE VIII. Percentage average total pretest and post-test scores (matched pairs) divided into three groups according to the pretest performance for students who did not work on the tutorial (nontutorial or comparison group) and only had lecture-based instruction in relevant content. The symbol $N$ denotes the total number of students in each range who took both the pretest and post-tests.

\begin{tabular}{lccc}
\hline \hline Pretest range $(\%)$ & $N$ & Pre & Post \\
\hline All & 57 & $38 \%$ & $55 \%$ \\
$0 \%-34 \%$ & 27 & $22 \%$ & $39 \%$ \\
$34 \%-67 \%$ & 29 & $51 \%$ & $68 \%$ \\
$67 \%-100 \%$ & 1 & $70 \%$ & $90 \%$ \\
\hline \hline
\end{tabular}

Table VII shows the performance of students on the pretest and post-tests partitioned into three separate groups based upon the performance on the pretest (see the range column). There are very new students in the highest pretest range. As can be seen from Table VII, the tutorial generally helped students both in the low and medium pretest range. Table VIII shows the performance of students in the comparison group (nontutorial group) on the pretest and post-tests partitioned into three separate groups based upon the pretest performance. As can be seen from comparing Tables VII and VIII, students in the comparison group did not perform on-par with those in the tutorial group on the post-test for the low and medium pretest ranges.

\section{SUMMARY}

We investigated the difficulties of students in a university introductory physics course with the electric field and superposition principle for the case of continuous charge distributions and used that research as a guide in the development, validation, and evaluation of a researchvalidated tutorial on these topics to help students learn these concepts. Some of the common difficulties that made the case of continuous charge distribution challenging for students include difficulties in applying the principle of superposition for the electric field and in generalizing from the discrete to the continuous charge distribution case, not recognizing the impact of the symmetry of the charge distribution on the electric field due to various continuous charge distributions, and assuming that the electric field would be radial or straight out from a continuous charge distribution even if the charge distribution did not have sufficient symmetry to justify it. The tutorial takes into account these common difficulties as a guide and uses a guided inquiry-based approach to learning and involved an iterative process of development and validation. In particular, the common difficulties are explicitly brought out and then the students are provided guidance and scaffolding support to build on their prior knowledge and develop good grasp of these challenging concepts. The final version of the validated tutorial was administered in several sections of the introductory physics course after traditional lecturebased instruction in relevant concepts. We compared the performance of students in individual interviews and on the pretest administered before the tutorial (but after traditional lecture-based instruction) and on the post-test administered after the tutorial in the introductory physics course. We also compared student performance in the sections of the course in which students worked on the tutorial with another section in which students only learned the materials via traditional lecture-based instruction. The data from the pretest and post-tests suggest that the tutorial was effective in improving student understanding of these concepts involving electric field due to a continuous charge distribution. Students performed significantly better in the sections of the course in which the tutorial was used compared to those who learned the content primarily via traditional lecture-based instruction. Moreover, the tutorial appears to be helpful for students who obtained low or medium scores on the pretest after traditional instruction.

\section{ACKNOWLEDGMENTS}

We would like to thank the National Science Foundation for Grant No. DUE-152457. We thank Z. Isvan for help in grading and tabulating the pretest and post-test data and thank F. Reif, P. Reilly, and R. P. Devaty for helpful discussions. We thank all faculty and students who helped in this study.

\section{APPENDIX: PRETEST AND POST-TESTS}

PRE: Assume all insulators (non-conductors) are nonpolarizable.

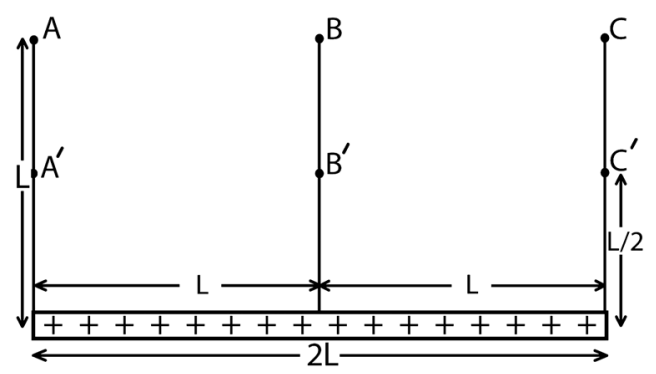

FIG. 5. A schematic diagram for the pretest questions (1) and (2). 
Infinitely long uniformly charged non-conducting solid cylinder
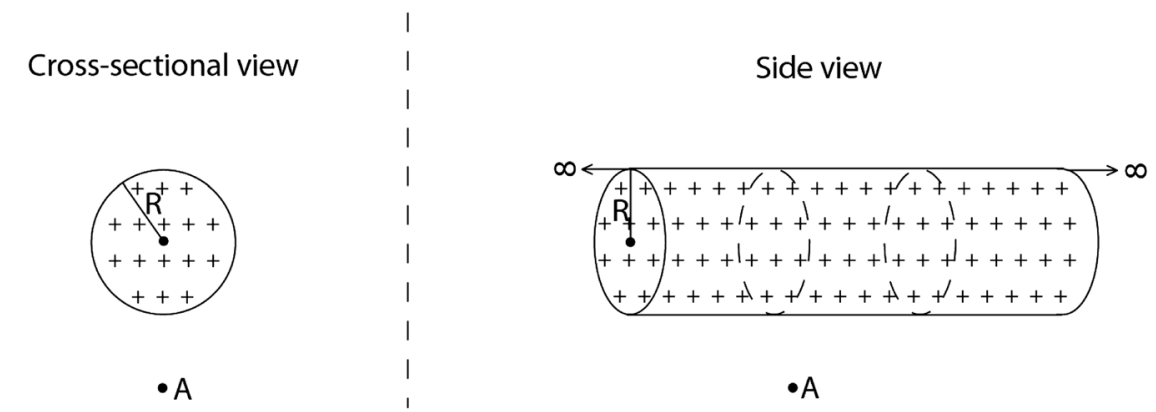

FIG. 6. A schematic diagram for the pretest question (3).

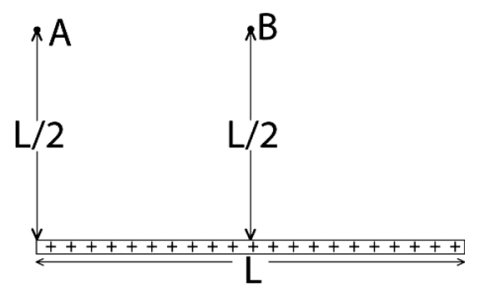

FIG. 7. A schematic diagram for the post-test question (1).

(1) Consider the three points $A, B$, and $C$ shown in Fig. 5, each at a distance $L$ above a finite uniform line of positive charge of length $2 L$. Draw arrows to show the direction of the net electric field at each of these three points. Explain.

(2) Consider the three points $A^{\prime}, B^{\prime}$, and $C^{\prime}$ shown in Fig. 5, each at a distance $L / 2$ above the finite uniform line of charge. Draw arrows to show the direction of the net electric field at each of these three points. Explain how the direction at point $A$ compares with $A^{\prime}$ and the direction at point $B$ compares with $B^{\prime}$.

(3) Shown in Fig. 6 are two views of an infinitely long solid cylinder of radius $\mathrm{R}$ with uniform charge distributed throughout its volume with charge per unit length $+\lambda$. On the cross-sectional view in Fig. 6, draw all points in the plane of the paper at which the net electric field has the same magnitude as at point $A$. Explain.
(4) On the cross-sectional view in Fig. 6, draw arrows to show the direction of the net electric field at four of the points that have the same magnitude of the net electric field as point $A$.

POST: (1) Consider the following statement from Susan about the electric field due to a finite line of length $\mathrm{L}$ with a uniformly distributed charge (see Fig. 7): "The field at a distance $L / 2$ above the line at both points $A$ and $B$ is directed perpendicular to the line of charge." Give a convincing argument that either supports her statement or refutes it.

(2) Imagine two concentric infinitely long hollow plastic cylinders of radii $R_{1}$ and $R_{2}$ with uniform surface charge (see Fig. 8). The linear charge densities are $-\lambda_{1}$ and $-\lambda_{2}$, respectively. On the cross-sectional view in Fig. 8, draw all points in the plane of the paper at which the net electric field has the same magnitude as at point $A$. Explain. Draw the directions of the net electric field at four of those points.

(3) What is the shape of the three-dimensional imaginary surface formed by all points that have the same magnitude of the net electric field as point $A$ in question (2)?

(4) Consider a square sheet of length $L$ on each side on which positive charge is uniformly distributed with a charge per unit area $\sigma$ (surface charge density). Consider two points, each at a height $h=L / 2$ above the sheet:

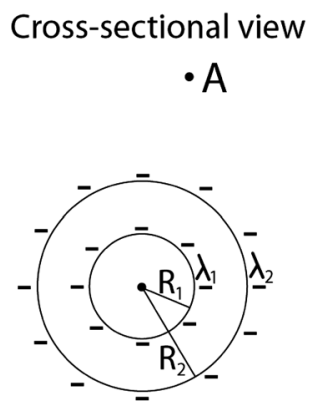

Side view

$\cdot A$

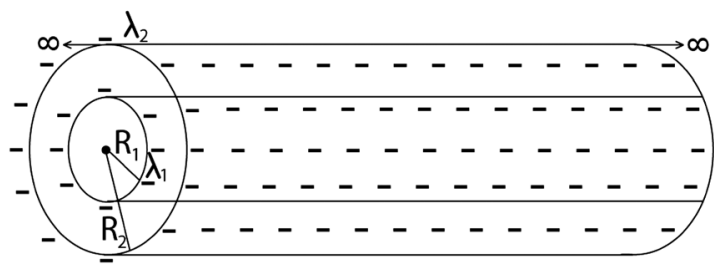

FIG. 8. A schematic diagram for the post-test questions (2) and (3). 
point $C$ is directly above the center of the sheet and point $B$ is off center (see Fig. 9). What, if anything, can you say about the directions of the net electric field at points $B$ and $C$ ? Explain. Are the magnitudes of the net electric field at points $B$ and $C$ equal? Explain.

(5) Consider the limit as the length of each side in the preceding question goes to infinity $(L \rightarrow \infty)$. What if anything, can you say about the direction of the net electric field at points $B$ and $C$ ? Explain. Are the magnitudes of the net electric field at points $B$ and $C$ equal? Explain.

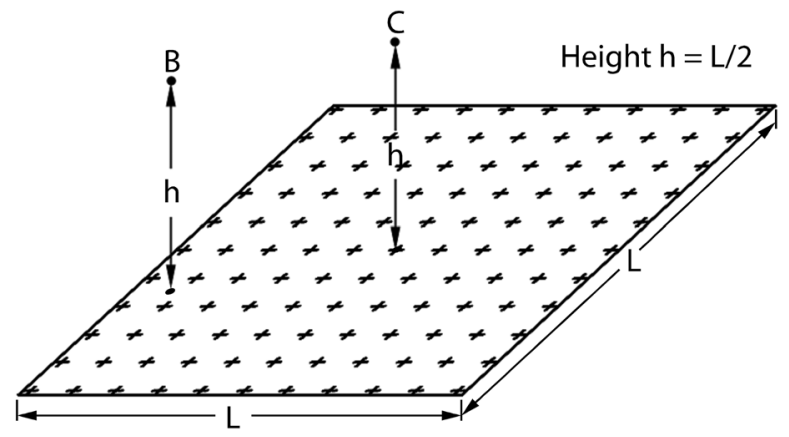

FIG. 9. A schematic diagram for the post-test question (4).
[1] B. Eylon and F. Reif, Effects of knowledge organization on task performance, Cognit. Instr. 1, 5 (1984).

[2] F. Reif, Millikan Lecture 1994: Understanding and teaching important scientific thought processes, Am. J. Phys. 63, 17 (1995).

[3] E. F. Redish, The Implications of Cognitive Studies for Teaching Physics, Am. J. Phys. 62, 796 (1994).

[4] D. Hammer, More than misconceptions: Multiple perspectives on student knowledge and reasoning, and an appropriate role for education research, Am. J. Phys. 64, 1316 (1996).

[5] D. Hu and N. Sanjay Rebello, Using Conceptual Blending to Describe How Students Use Mathematical Integrals in Physics, Phys. Rev. ST Phys. Educ. Res. 9, 020118 (2013).

[6] D. Hu and N. Sanjay Rebello, Understanding Student Use of Differentials in Physics Integration Problems, Phys. Rev. ST Phys. Educ. Res. 9, 020108 (2013).

[7] D. Zollman, Oersted Lecture 2014: Physics education research and teaching modern physics, Am. J. Phys. 84, 573 (2016).

[8] D. Meltzer, Relation between students' problem solving performance and representational mode, Am. J. Phys. 73, 463 (2005).

[9] C. Singh, When physical intuition fails, Am. J. Phys. 70, 1103 (2002).

[10] C. Singh, Assessing student expertise in introductory physics with isomorphic problems. I. Performance on a nonintuitive problem pair from introductory physics, Phys. Rev. ST Phys. Educ. Res. 4, 010104 (2008).

[11] C. Singh, Assessing student expertise in introductory physics with isomorphic problems. II. Effect of some potential factors on problem solving and transfer, Phys. Rev. ST Phys. Educ. Res. 4, 010105 (2008).

[12] S. Y. Lin and C. Singh, Effect of scaffolding on helping introductory physics students solve quantitative problems involving strong alternative conceptions, Phys. Rev. ST Phys. Educ. Res. 11, 020105 (2015).

[13] S. Y. Lin and C. Singh, Using isomorphic problem pair to learn introductory physics: Transferring from a two-step problem to a three-step problem, Phys. Rev. ST Phys. Educ. Res. 9, 020114 (2013).
[14] S. Y. Lin and C. Singh, Using isomorphic problems to learn introductory physics, Phys. Rev. ST Phys. Educ. Res. 7, 020104 (2011).

[15] S. Y. Lin and C. Singh, Challenges in using analogies, Phys. Teach. 49, 512 (2011).

[16] L. McDermott and P. Shaffer (The Physics Education Group), Tutorials in Introductory Physics (Prentice Hall, Englewood Cliffs, NJ, 2002).

[17] L. McDermott and P. Shaffer, Research as a guide for curriculum development: An example from introductory electricity. Part I: Investigation of student understanding, Am. J. Phys. 60, 994 (1992).

[18] L. McDermott and P. Shaffer, Research as a guide for curriculum development: An example from introductory electricity. Part II: Design of instructional strategies, Am. J. Phys. 60, 994 (1992).

[19] B. Eylon and U. Ganiel, Macro-micro relationships: The missing link between electrostatics and electrodynamics in students' reasoning, Int. J. Sci. Educ. 12, 79 (1990).

[20] G. Gladding, M. Selen, and T. Stelzer, Electricity and Magnetism, https://www.amazon.com/ElectricityMagnetism-SmartPhysics-Gary-Gladding/dp/1429272392.

[21] K. Zuza, M. De Cock, P. van Kampen, L. Bollen, and J. Guisasola, University students' understanding of the electromotive force concept in the context of electromagnetic induction, Eur. J. Phys. 37, 065709 (2016).

[22] I. Garzón, M. De Cock, K. Zuza, P. van Kampen, and J. Guisasola, Probing university students' understanding of electromotive force in electricity, Am. J. Phys. 82, 72 (2014).

[23] C. Guruswamy, M. Somers, and R. Hussey, Students' understanding of transfer of charge between conductors, Phys. Educ. 32, 91 (1997).

[24] J. Guisasola, J. Zubimendi, J. Almudi, and M. Ceberio, The evolution of the concept of capacitance throughout the development of the electric theory and the understanding of its meaning by university students, Sci. Educ. 11, 247 (2002).

[25] J. Guisasola, J. Zubimendi, and K. Zuza, How much have students learned? Research-based teaching on electrical capacitance, Phys. Rev. ST Phys. Educ. Res. 6, 020102 (2010). 
[26] K. Zuza, M. De Cock, P. van Kampen, L. Bollen, and J. Guisasola, University students' understanding of the electromotive force concept in the context of electromagnetic induction, Eur. J. Phys. 37, 065709 (2016).

[27] E. Dębowska, R. Girwidz, T. Greczyło, A. Kohnle, B. Mason, L. Mathelitsch, T. Melder et al., Report and recommendations on multimedia materials for teaching and learning electricity and magnetism, Eur. J. Phys. 34, L47 (2013).

[28] E. R. Savelsbergh, Ton de Jong, and M. G. FergusonHessler, Choosing the right solution approach: The crucial role of situational knowledge in electricity and magnetism, Phys. Rev. ST Phys. Educ. Res. 7, 010103 (2011).

[29] T. Sujarittham, N. Emarat, K. Arayathanitkul, M. Sharma, I. Johnston, and J. Tanamatayarat, Developing specialized guided worksheets for active learning in physics lectures, Eur. J. Phys. 37, 025701 (2016).

[30] C. Efthimiou, D. Maronde, T. McGreevy, E. del Barco, and S. McCole, Implementing elements of The Physics Suite at a large metropolitan research university, Phys. Educ. 46, 421 (2011).

[31] J. Lenaerts, W. Wieme, and E. Van Zele, Peer instruction: A case study for an introductory magnetism course, Eur. J. Phys. 24, 7 (2003).

[32] S. F. Itza-Ortiz, S. Rebello, and D. Zollman, Students' models of Newton's second law in mechanics and electromagnetism, Eur. J. Phys. 25, 81 (2004).

[33] G. Alejandro and G. Zavala, Contrasting students' understanding of electric field and electric force, AIP Conf. Proc. 1513, 142 (2013).

[34] D. Sokoloff and R. Thornton, Using interactive lecture demonstrations to create an active learning environment, Phys. Teach. 35, 340 (1997).

[35] B. Thacker, U. Ganiel, and D. Boys, Macroscopic phenomena and microscopic processes: Student understanding of transients in direct current electric circuits, Am. J. Phys. 67, S2 (1999).

[36] S. Y. Lin, A. Maries, and C. Singh, Student difficulties in translating between mathematical and graphical representations in introductory physics, AIP Conf. Proc. 1513, 250 (2013).

[37] J. Li and C. Singh, Students' difficulties with equations involving circuit elements, AIP Conf. Proc. 1413, 243 (2012).

[38] J. Li and C. Singh, Students' common difficulties and approaches while solving conceptual problems with nonidentical light bulbs in series and parallel, Eur. J. Phys. 37, 065708 (2016).

[39] J. Bilak and C. Singh, Improving students' conceptual understanding of conductors and insulators, AIP Conf. Proc. 951, 49 (2007).

[40] Z. Isvan and C. Singh, Improving student understanding of Coulomb's law and Gauss's law, AIP Conf. Proc. 883, 181 (2007).

[41] A. Maries and C. Singh, Should students be provided diagrams or asked to draw them while solving introductory physics problems?, AIP Conf. Proc. 1413, 263 (2012).

[42] A. Maries and C. Singh, To use or not to use diagrams: The effect of drawing a diagram in solving introductory physics problems, AIP Conf. Proc. 1513, 282 (2013).
[43] A. Maries, S. Y. Lin, and C. Singh, Challenges in designing appropriate scaffolding to improve students' representational consistency: The case of a Gauss's law problem, Phys. Rev. Phys. Educ. Res. 13, 020103 (2017).

[44] C. Singh, Impact of peer interaction on conceptual test performance, Am. J. Phys. 73, 446 (2005).

[45] A. Maries and C. Singh, Do students benefit from drawing productive diagrams themselves while solving introductory physics problems? The case of two electrostatics problems, Eur. J. Phys. 39, 015703 (2018).

[46] A. Maries and C. Singh, Case of two electrostatics problems: Can providing a diagram adversely impact introductory physics students' problem solving performance?, Phys. Rev. Phys. Educ. Res. 14, 010114 (2018).

[47] C. Singh, Effectiveness of group interaction on conceptual standardized test performance, Proceedings of the Physics Education Research Conference, Boise, Idaho (AIP, New York, 2002), p. 67, DOI: 10.1119/perc.2002.pr.017.

[48] J. Li and C. Singh, Investigating and improving introductory physics students' understanding of the electric field and superposition principle, Eur. J. Phys. 38, 055702 (2017).

[49] J. Li and C. Singh, Investigating and improving introductory physics students' understanding of electric flux, Eur. J. Phys. 39, 045711 (2018).

[50] J. Li and C. Singh, Investigating and improving introductory physics students' understanding of symmetry and Gauss's law, Eur. J. Phys. 39, 015702 (2018).

[51] E. Mazur, Peer Instruction: A User's Manual, Prentice Hall Series in Educational Innovation (Prentice Hall, Upper Saddle River, NJ, 1997).

[52] D. Maloney, T. O'Kuma, C. Hieggelke, and A. Van Heuvelen, Surveying students' conceptual knowledge of electricity and magnetism, Am. J. Phys. 69, S12 (2001).

[53] L. Ding, R. Chabay, B. Sherwood, and R. Beichner, Valuating an assessment tool: Brief Electricity and Magnetism Assessment, Phys. Rev. ST Phys. Educ. Res. 2, 010105 (2006).

[54] P. Engelhardt and R. Beichner, Students' understanding of direct current resistive electrical circuits, Am. J. Phys. 72, 98 (2004).

[55] C. Singh, Improving students' understanding of magnetism, in Proceedings of the 2008 American Society for Engineering Education Annual Conference \& Exposition, Pittsburgh, Pennsylvania (2008), pp. 1-16, https://peer .asee.org/improving-students-understanding-of-magnetism.

[56] J. Li and C. Singh, Developing a magnetism conceptual survey and assessing gender differences in student understanding of magnetism, AIP Conf. Proc. 1413, 43 (2012).

[57] J. Li and C. Singh, Developing and validating a conceptual survey to assess introductory physics students' understanding of magnetism, Eur. J. Phys. 38, 025702 (2017).

[58] C. Singh, Student understanding of symmetry and Gauss's law of electricity, Am. J. Phys. 74, 923 (2006).

[59] C. Singh, Student understanding of symmetry and Gauss's Law, AIP Conf. Proc. 790, 65 (2005).

[60] J. McMillan and J. Wergin, Understanding and Evaluating Educational Research, 4th ed. (Pearson, Prentice Hall, Englewood Cliffs, NJ, 2010). 
[61] B. Kirwan and L. Ainsworth, A Guide to Task Analysis (Taylor \& Francis, London, 1992).

[62] R. E. Clark, D. F. Feldon, J. G. van Merriënboer, K. Yates, and S. Early, Cognitive task analysis, in Handbook of Research on Educational Communications and
Technology, 3rd ed., edited by J. M. Spector, M. D. Merrill, J. G. van Merriënboer, and M. P. Driscoll (Lawrence Erlbaum Associates, Mahwah, NJ, 2007).

[63] https://drive.google.com/file/d/1V0iwObrL1RSiM9yD2A hnrPF6aQx4FEe9/view. 